\title{
La violencia contra las mujeres en Colombia, un desafío para la salud pública en cuanto a su prevención, atención y eliminación
}

Violence against women in Colombia, a challenge for public health in regard to its prevention, care and elimination

\author{
Elizabeth García Restrepo ${ }^{1 \otimes 0 R C I D}$ \\ Doris Cardona 2 ORCID \\ Andrés Felipe Tirado Otálvaro ${ }^{3} \underline{\mathrm{ORCID}}$
}

Fecha correspondencia:

Recibido: 25 de noviembre de 2020. Revisión: 16 de diciembre de 2020. Aceptado: 25 de enero de 2021.

\section{Forma de citar:}

García, Elizabeth; Cardona, Doris;

Tirado, Andrés Felipe. (2021). "La

violencia contra las mujeres en

Colombia, un desafío para la salud pública en cuanto a su prevención, atención y eliminación". En: Revista CES Derecho. Vol. 12, №. 1, enero a junio de 2021, 167-175.

Open access

Términos de uso

Licencia creative commons

Etica de publicaciones

Revisión por pares

Gestión por Open Journal System

DOl: https://dx.doi.org/10.21615/

cesder.12.1.9

ISSN: 2145-7719

Sobre los autores:

1. Elízabeth García Restrepo.

Psicóloga, Especialista en Gerencia de la Salud Pública, estudiante del Doctorado en Salud Pública de la Escuela de Graduados de la Universidad CES.

2. Doris Cardona Arango.

\section{Resumen}

La salud pública como campo de conocimiento, práctica e investigación tiene mucho que aportar al tema de la violencia contra las mujeres, reconocido como problema global y local hace relativamente poco tiempo; puede contribuir a hacer evidente la magnitud del problema y sus consecuencias, ayudar a dilucidar sus causas, diseñar políticas públicas para la prevención e intervención oportuna; la gestión del conocimiento; la promoción de la salud y la integración de los saberes de las organizaciones y las comunidades, como medio para su transformación. En este ensayo desde una mirada de la salud pública, se hace un recorrido por este problema en Colombia, mostrando algunos de estos alcances, retos y oportunidades.

Palabras claves: violencia contra la mujer, mujeres maltratadas, violencia de género, salud pública, Colombia.

\section{Abstract}

Public health as a field of knowledge, practice, and research has much to contribute to the issue of violence against women, recognized as a global and local problem relatively recently; It can contribute to make evident the magnitude of the problem and its consequences, help to elucidate its causes, design public policies for prevention and timely intervention; knowledge management; the promotion of health and the integration of the knowledge of organizations and communities, as a means for their transformation. In this essay from a public health perspective, a tour of this problem in Colombia is made, showing some of these scopes, challenges and opportunities.

Keywords: violence against women, battered women, gender-based violence, public health, Colombia. 
Administradora de

empresas, Magister en epidemiología, Magister en Salud Pública, Doctora en Demografía. Coordinadora de la Maestría en Salud Pública de la Universidad CES.

3. Andrés Felipe Tirado Otálvaro. Enfermero Magíster en Epidemiología, Doctor en Salud Pública. Profesor titular, Facultad de Enfermería Universidad Pontificia Bolivariana, Grupo de Investigación en Cuidado.

\section{Introducción}

La Convención Interamericana para Prevenir, Sancionar y Erradicar la Violencia contra la Mujer, ratificada por el Estado Colombiano, mediante la Ley 248 de 1995 (Ley 248 de 1995. Publicada en el Diario Oficial No. 42.171, de diciembre 29 de 1995, s. f.) define por violencia contra la mujer "...cualquier acción o conducta basada en su género, que cause muerte, daño o sufrimiento físico, sexual o psicológico a la mujer, tanto en el ámbito público como en el privado". Reconoce tres tipos de violencia: física, sexual y psicológica y tres ámbitos donde se manifiesta: 1) La vida privada, cuando la violencia se ejerce dentro de la familia, la unidad doméstica o en cualquier otra relación interpersonal, aun cuando el agresor ya no viva con la mujer; 2) La vida pública, cuando la violencia es ejercida por cualquier persona, ya sea que esta se lleve a cabo en la comunidad, en el lugar de trabajo, en instituciones educativas, establecimientos de salud o en cualquier otro lugar; y 3) La perpetrada o tolerada por el Estado o sus agentes, donde quiera que ocurra (Organización de Estados Americanos, OEA, 1994).

Del mismo modo, establece cinco premisas rectoras: 1) Constituye una violación de los derechos humanos y las libertades fundamentales; 2) Es una ofensa a la dignidad humana y una manifestación de las relaciones de poder históricamente desiguales entre mujeres y hombres; 3) Transciende todos los sectores sociales, es un problema universal de proporciones epidémicas, independientemente de la clase, el grupo étnico, el nivel de ingresos o educativo, la cultura, la edad o la religión, aunque las manifestaciones varíen en el contexto social, económico e histórico; 4) la eliminación de la violencia, es condición para el desarrollo igualitario, y 5) Reconoce expresamente el derecho a una vida libre de violencia, tanto en el ámbito público como en el privado (Organización de Estados Americanos, OEA, 1994).

La violencia contra las mujeres, se ha nombrado de distintas formas dependiendo de diversos marcos interpretativos (Gómez C et al., 2013), que hacen un énfasis en particular, por ejemplo, desde el lugar o el escenario donde se presenta (violencia en el hogar, violencia doméstica); entre quienes ocurre (violencia intrafamiliar, violencia de pareja íntima) y las relaciones de poder (violencia de género); este último término, hace hincapié en explicitar que dicha violencia, es una manifestación de relaciones desiguales entre hombres y mujeres, establecidas por condicionamientos sociales y culturales, en el caso de la violencia de género hacia las mujeres, por su condición de serlo (Vives C, 2011); sin embargo, dicho concepto también abarca la violencia que se presenta contra las personas que tienen una orientación sexual e identidad de género no heteronormativa (Ortiz M et al., 2015), es decir, que tienen una orientación sexual distinta a la heterosexual, que ha sido la norma socialmente establecida (Aller L, 1995). Para efectos de este texto, por violencia contra la mujer se empleará la definición propuesta desde el marco internacional que adopta la legislación colombiana: "cualquier acción u omisión, que le cause muerte, daño o sufrimiento físico, sexual, psicológico, económico o patrimonial por su condición de mujer, así como las amenazas de tales actos, la coacción o la privación arbitraria de la libertad, bien sea que se presente en el ámbito público o en el privado" (Ley 1257 de 2008, 2008), reconociendo que dicha violencia, es producto de relaciones históricamente desiguales entre hombres y mujeres (Organización de Estados Americanos, OEA, 1994).

Con relación a las cifras y las expresiones de la violencia contra las mujeres en Colombia, de acuerdo con la Encuesta Nacional de Demografía y Salud del 2015, el $64,1 \%$ de las mujeres manifestó ser víctima de violencia psicológica, el 31,6\% de violencia física, el 31\% víctima de violencia económica y el 7,6\% víctima de violencia sexual (Ministerio de Salud Protección Social - Colombia \& Asociación Probienestar 
de la Familia Colombiana (Profamilia) - Colombia, 2015). Por su parte, de acuerdo con el Instituto Nacional de Medicina Legal y Ciencias Forenses (Instituto Nacional de Medicina Legal y Ciencias Forenses, 2020), durante el año 2019 se presentaron 77.303 casos de violencia intrafamiliar, $76,2 \%$ en mujeres. En cuanto a los exámenes médico legales por presunto delito sexual se registraron 26.158, una tasa de 52,9 por 100.000 habitantes; según el sexo de la víctima el 84,9\% eran mujeres. Al revisar las cifras de homicidios de mujeres para este mismo año, se reportan 1.001 casos, una tasa de 2,0 por 100.000 habitantes (Instituto Nacional de Medicina Legal y Ciencias Forenses, 2020). Por otra parte, al hacer un análisis del fenómeno en el ámbito específico del conflicto armado en Colombia, la Unidad para la Atención y Reparación a Víctimas (UARIV), en el Registro Único de Víctimas (RUV), a la consulta entre 1985 y el 01 de enero de 2020 se reporta un total de 8.553 .416 personas afectadas por hechos victimizantes. De este total 4.277 .995 son mujeres (Unidad para la Atención y Reparación Integral a las Victimas, 2020). Si bien estas cifras son alarmantes y develan que la violencia contra las mujeres es un problema de salud pública en el país, es importante considerar, entre otras, el subregistro que se presenta, debido a que se denuncian menos hechos de los que realmente suceden (Grupo Centro de Referencia Nacional sobre Violencia, 2015), lo cual significa que los datos oficiales podrían ser tan solo la punta del iceberg y que no se ha logrado caracterizar en toda su real dimensión.

En consideración con lo anterior, uno de los desafíos que enfrenta Colombia y particularmente la salud pública, es contribuir con la eliminación de todas las formas de violencia contra las mujeres y las niñas como parte de los objetivos de la igualdad de género, considerada la base necesaria para conseguir un mundo pacífico, próspero y sostenible, una de las metas en la Agenda 2030 para el Desarrollo Sostenible (United Nations, s. f.), que nos reta como academia y sociedad. En tal sentido, en este ensayo se hace un recorrido teórico desde la salud pública por este problema en el país, con el objetivo de aportar a la discusión y a la acción, como una forma de contribuir a su prevención, atención y a la apuesta, por su eliminación.

\section{La violencia contra las mujeres, una mirada desde la salud pública}

La Asamblea Mundial de la Salud, declaró la violencia como uno de los principales problemas de salud pública en todo el mundo y resaltó sus graves consecuencias a corto y largo plazo para los individuos, las familias, las comunidades, los países y los servicios de salud e instó a los Estados Miembros a que evaluaran el problema en sus territorios, caracterizaran los distintos tipos, se definiera su magnitud, incorporando en el análisis una perspectiva basada en las diferencias entre los géneros y se promovieran investigaciones desde la salud pública (13).

Una de sus formas de expresión, es la violencia contra las mujeres, reconocida por la Organización Mundial de la Salud (OMS), como un problema grave de salud pública, por su magnitud y por las consecuencias que genera en el bienestar físico y psicológico de las mujeres, las familias y la sociedad en su conjunto (Krug E et al., 2002). Algunas de sus consecuencias mortales, documentadas en distintas investigaciones internacionales y nacionales son los homicidios, feminicidios, suicidios (Hernández W, 2015; Karlsson et al., 2019; Sierra S et al., 2018), infección por VIH/SIDA, cáncer; así como también mortalidad materna (Aygin \& Bozdemir, 2019; Baigorria et al., 2017; Krug E et al., 2002). En las consecuencias no mortales se ha reportado discapacidad (Aygin \& Bozdemir, 2019), trastornos mentales relacionados con estrés postraumático, 
depresión, ansiedad y consumo de sustancias psicoactivas (Baigorria et al., 2017; Mittal et al., 2018); al igual que problemáticas en la salud sexual y la salud reproductiva, como embarazos no planeados y abortos (Ely \& Murshid, 2018; Krug E et al., 2002). Desde otro punto de vista, la violencia contra las mujeres genera impactos económicos y sociales (Asamblea General Naciones Unidas, 2006; Krug E et al., 2002) y desde el enfoque de derechos, constituye una violación a las libertades fundamentales y a los derechos humanos de las mujeres (Asamblea General Naciones Unidas, 2006; Organización de Estados Americanos, OEA, 1994).

Su concepción como problema en la salud pública es relativamente reciente, pues hasta la década de 1980 era entendido como un asunto personal (García et al., 2017); en el plano internacional por la incidencia política de las organizaciones de mujeres sólo hasta 1993 se reconoce la discriminación y violencia contra las mujeres, como una violación a sus derechos humanos (Declaración y Programa de Acción de Viena, s. f.) y a partir de 1995 los gobiernos del mundo expresan la determinación de garantizar su eliminación a través de la Plataforma de Acción de Beijing, como una de las formas para hacer posible el desarrollo sostenible y equitativo (Naciones Unidas, 1995). En este sentido, el sector salud liderado por la OMS/OPS empieza a hacerlo visible como un problema de salud pública a nivel global y local (García C et al., 2015). Para ello se han retomado los enfoques y métodos que la salud pública ha desarrollado para explicar y comprender la violencia en general, uno de los cuales es el modelo ecológico, propuesto en los años 70's del siglo XX (Bronfenbrenner, 1996), el sistema de vigilancia en salud pública, a través del cual se recopila, analiza, interpreta de manera sistemática y continua los datos para la generación de acciones que beneficien la salud de las poblaciones (Groseclose \& Buckeridge, 2017), en este caso para la prevención y el control de la violencia y la formulación de las políticas públicas (Krug E et al., 2002); además, para la intervención se ha recurrido al diseño de estrategias basadas en distintos niveles de prevención, de acuerdo con el momento en que se intervenga la violencia, sin embargo, desde el sector salud todavía su abordaje integral es incipiente (Ortiz G \& Vives C, 2012).

Para complementar la discusión desde otras aristas en salud pública, se exponen dos elementos fundamentales, la complejidad y multicausalidad del problema y la concepción de salud desde la cual se parte para su intervención. Con relación al primer punto, se han planteado diferentes causas, factores de riesgo y modelos explicativos para la comprensión e interpretación de la violencia contra las mujeres, entre ellos, enfoques individuales centrados en las características de las víctimas y los agresores; miradas que se enfocan en las relaciones de poder entre hombres y mujeres; en los factores estructurales y simbólicos desde lo macrosocial y el análisis de interseccionalidad (Jiménez ML \& Guzmán R, 2015). Sin embargo, uno de los enfoques que se considera más integral porque recoge distintas perspectivas multicausales y feministas que se ha aplicado en la investigación en Colombia desde ONU Mujeres (Fondo de Desarrollo de las Naciones Unidas para la Mujer, UNIFEM, 2010), es el modelo propuesto por Heise Lori (Heise, L, 1998), el cual partiendo del modelo ecológico (Bronfenbrenner, 1996), reconoce la violencia contra las mujeres como un fenómeno dinámico que se genera y reproduce en la interacción de cuatro ámbitos: las historias personales (endosistema), las relaciones más inmediatas (microsistema), los ámbitos socioeconómicos donde se producen (exosistema) y los contextos culturales (macrosistemas), incorporando tanto el análisis de los atributos de las víctimas y de los agresores, como los contextos donde la acción violenta se produce (Fondo de Desarrollo de las Naciones Unidas para la Mujer, UNIFEM, 2010). 
Por otro lado, el segundo punto para la discusión, que desde el sector salud limita el abordaje integral de la violencia contra las mujeres, es la concepción misma de la salud de la cual se parte para la comprensión del problema y para su efectivo tratamiento; en el modelo biomédico dominante en la salud pública tradicional, en la que prima el presupuesto filosófico y teórico de la enfermedad y la muerte (Granda E, 2008), se ha orientado el problema principalmente hacia la atención de las mujeres víctimas cuando llegan al sistema de salud, por ejemplo a los servicios de urgencia, a la consulta clínica; y no a la prevención y a la transformación de sus raíces. Con respecto al método que se ha utilizado, si bien la epidemiología clásica contribuye a la explicación de los factores de riesgo para la violencia contra las mujeres, es insuficiente para la comprensión y tratamiento integral del problema (Ruiz P et al., 2004). Por otro lado, el Estado y mucho menos el sector salud actuando solos pueden prevenir y atender, la violencia contra las mujeres y lograr promover nuevas formas de relaciones humanas, sino cuentan con otros actores y sectores estratégicos. Como se plantea desde la evidencia científica, es necesario recurrir a un enfoque multisectorial y de acciones multinivel, en una apuesta de intervención a largo plazo, articulada y continua que incluya el abordaje de las estructuras sociales, culturales, económicas y políticas que generan la discriminación y las inequidades hacia las mujeres (Ellsberg et al., 2015; García C et al., 2015). Desde el punto de vista de la salud pública latinoamericana, un enfoque emancipatorio y de acción colectiva desde la promoción de la salud, como la propuesta por Chapela y García (Chapela MC \& Cerda A, 2010), donde es fundamental incorporar los conocimientos y recursos populares para el entendimiento y la solución de los problemas de salud, las comunidades y las organizaciones de mujeres tienen mucho que aportar para la solución del problema, desde su capacidad de agencia, exigibilidad de derechos y reconocimiento como promotoras de la salud; de hecho gracias a sus esfuerzos, el problema se hizo visible y se mantiene vigente en la agenda política internacional y nacional (Naciones Unidas, 1995; Declaración y Programa de Acción de Viena, s. f.).

Retomando el modelo de Lori aplicado al contexto colombiano, desde lo que se ha documentado en los resultados de las investigaciones de ONU Mujeres en el país (Fondo de Desarrollo de las Naciones Unidas para la Mujer, UNIFEM, 2010) y lo que otras evidencias científicas plantean (Ellsberg et al., 2015; García C et al., 2015; Velzeboer $\mathrm{M}$ et al., 2003), para lograr prevenir, atender y erradicar las violencias contra las mujeres en Colombia, sería necesario, intervenir y modificar cada uno de los cuatro ámbitos descritos, en una apuesta de intervención a largo plazo, articulada y continua en la que participen diferentes actores y sectores estratégicos, entre ellos, el Estado, con su institucionalidad, la sociedad civil representada entre otras, por organizaciones de mujeres y de base comunitaria, la academia, organizaciones no gubernamentales, el sector privado, haciendo un abordaje de las estructuras sociales, culturales, económicas y políticas que generan la discriminación y las inequidades hacia las mujeres para logar impactar la multicausalidad y complejidad del problema.

\section{Conclusiones}

La violencia contra las mujeres es un problema complejo, multifactorial y con distintas formas de expresión que desde la salud pública se puede contribuir a prevenir, atender y eliminar, para lo cual se necesitan respuestas diferenciales y complementarias con otras disciplinas y saberes sociales que permitan desentrañar las raíces y prácticas que la han naturalizado, sustentadas en relaciones de poder históricamente desiguales entre los hombres y las mujeres, que se refleja en la vida pública y privada. 
El enfoque de la violencia contra las mujeres parte de una mirada tradicional de la salud pública en la que principalmente se identifican y se busca intervenir algunos factores de riesgo; sin embargo, es necesario avanzar, e integrar en la mirada otras perspectivas que, desde la vida, la promoción de la salud y la participación de las mujeres contribuyan a la transformación de este grave problema que afecta la salud de las poblaciones.

Promover la investigación y la gestión del conocimiento en salud pública para la problemática de la violencia contra las mujeres y el derecho a una vida libre de esta, es una manera de contribuir con la evidencia para orientar las políticas públicas en la materia.

\section{Agradecimientos}

A la Dirección de Investigación e Innovación de la Universidad CES, por el apoyo financiero para el desarrollo de la investigación "La construcción de paz desde las acciones colectivas de las organizaciones de mujeres y su contribución al derecho a una vida libre de violencia. Medellín 2000-2016", bajo la modalidad de mediana cuantía, código INV.032017.005.

\section{Bibliografía}

Aller L. (1995). Pedagogía de la sexualidad humana. Una aproximacón ideológica y metodológica. Galerna.

Asamblea General Naciones Unidas. (2006). Estudio a fondo sobre todas las formas de violencia contra la mujer Informe del Secretario General.

Aygin, D., \& Bozdemir, H. (2019). Exposure to violence in breast cancer patients: Systematic review. Breast Cancer (Tokyo, Japan), 26(1), 29-38. https://doi. org/10.1007/s12282-018-0900-6

Baigorria, J., Warmling, D., Magno Neves, C., Delziovo, C. R., \& Salema Coelho, E. B. (2017). [Prevalence and associated factors with sexual violence against women: Systematic review]. Revista De Salud Publica (Bogota, Colombia), 19(6), 818-826. https://doi.org/10.15446/rsap.V19n6.65499

Bronfenbrenner, U. (1996). The ecology of human development: Experiments by nature and design. Harvard University Press.

Chapela MC, \& Cerda A. (2010). Promoción de la salud y poder: Reformulaciones desde el cuerpo-territorio y la exigibilidad de derechos (Primera edición).

Ley 248 de 1995. Publicada en el Diario Oficial No. 42.171, de diciembre 29 de 1995, 9. https://www.defensoria.gov.co/public/Normograma\%202013 html/Normas/ Ley 248 1995.pdf

Ley 1257 de 2008, Diario Oficial 47193 de diciembre 4 de 2008 (2008). http://www. alcaldiabogota.gov.co/sisjur/normas/Norma1.jsp?i=34054

Ellsberg, M., Arango, D. J., Morton, M., Gennari, F., Kiplesund, S., Contreras, M., \& Watts, C. (2015). Prevention of violence against women and girls: What does the evidence say? The Lancet, 385(9977), 1555-1566. https://doi.org/10.1016/S01406736(14)61703-7 
Ely, G. E., \& Murshid, N. S. (2018). The Relationship Between Partner Violence and Number of Abortions in a National Sample of Abortion Patients. Violence and Victims, 33(4), 585-603. https://doi.org/10.1891/0886-6708.VV-D-16-00215

Fondo de Desarrollo de las Naciones Unidas para la Mujer, UNIFEM. (2010). Estudio sobre tolerancia social e institucional a la violencia basada en genero en Colombia. https://www.medellin.gov.co/irj/go/km/docs/wpccontent/Sites/Subportal\%20 del\%20Ciudadano/Equidad\%20de\%20G\%C3\%A9nero/Secciones/Informes/ Documentos/2011/Estudio Colombia.pdf

García C, Zimmerman, C., Morris-Gehring, A., Heise, L., Amin, A., Abrahams, N., Montoya, O., Bhate-Deosthali, P., Kilonzo, N., \& Watts, C. (2015). Addressing violence against women: A call to action. The Lancet, 385(9978), 1685-1695. https://doi.org/10.1016/ S0140-6736(14)61830-4

García, O., Alejandra, M., Melo, I., \& Eugenia, M. (2017). Detrás de las cifras de violencia contra las mujeres en Colombia. Sociedad y Economía, 32, 41-64.

Gómez C, Murad R, \& Calderón M. (2013). Historias de violencia, roles, prácticas y discursos legitimadores. Violencia contra las mujeres en Colombia 2000-2010. Profamilia.

Granda E. (2008). El saber en salud pública en un ámbito de pérdida de antropocentrismo y ante una visión de equilibrio ecológico. Revista Facultad Nacional de Salud Pública, 26, 65-90.

Groseclose, S. L., \& Buckeridge, D. L. (2017). Public Health Surveillance Systems: Recent Advances in Their Use and Evaluation. Annual Review of Public Health, 38(1), 57-79. https://doi.org/10.1146/annurev-publhealth-031816-044348

Grupo Centro de Referencia Nacional sobre Violencia. (2015). Masatugó 2009-2014. Forensis de Mujeres: Herramienta para la interpretación, intervención y prevención de lesiones de causa externa en Colombia para las mujeres (Instituto Nacional de Medicina Legal y Ciencias Forenses, Ed.). Diseñum Tremens. https://www.medicinalegal.gov.co/cifras-estadisticas/masatugo

Heise, L. (1998). Violence against women: An integrated, ecological framework. Violence against women., Vol. 4. No. 3., 262-290.

Hernández W. (2015). Feminicidio (agregado) en el Perú y su relación con variables macrosociales. URVIO - Revista Latinoamericana de Estudios de Seguridad, 17, 48-66. https://doi.org/10.17141/urvio.17.2015.2007

Instituto Nacional de Medicina Legal y Ciencias Forenses. (2020, octubre 19). Forensis 2019. Datos para la vida. Información de lesiones de causa externa y desaparecidos. Violencia intrafamiliar y homicidios. Colombia, 2019. https://www.medicinalegal. gov.co/cifras-estadisticas/forensis

Jiménez ML, \& Guzmán R. (2015). El caleidoscopio de la violencia contra las mujeres en la pareja desde la desigualdad de género: Una revisión de enfoques analíticos. Revista de Estudios Sociales, 54, 93-106. https://doi.org/10.7440/res54.2015.07 
Karlsson, L. C., Antfolk, J., Putkonen, H., Amon, S., da Silva Guerreiro, J., de Vogel, V., Flynn, S., \& Weizmann-Henelius, G. (2019). Familicide: A systematic literature review. Trauma, Violence \& Abuse, 1524838018821955. https://doi. org/10.1177/1524838018821955

Krug E, Dahlberg L, Mercy J, Zwi A, \& Lozano R (Eds.). (2002). World report on violence and health.

Ministerio de Salud Protección Social - Colombia, \& Asociación Probienestar de la Familia Colombiana (Profamilia) - Colombia. (2015). Encuesta Nacional de Demografía y Salud, 2015.

Mittal, M., Resch, K., Nichols-Hadeed, C., Thompson Stone, J., Thevenet-Morrison, K., Faurot, C., \& Cerulli, C. (2018). Examining Associations Between Strangulation and Depressive Symptoms in Women With Intimate Partner Violence Histories. Violence and Victims, 33(6), 1072-1087. https://doi.org/10.1891/0886$\underline{6708.33 .6 .1072}$

Declaración y Programa de Acción de Viena, 59. https://www.ohchr.org/Documents/ Events/OHCHR20/VDPA booklet Spanish.pdf

Naciones Unidas. (1995). Declaración y Plataforma de Acción de Beijing. http://www. un.org/womenwatch/daw/beijing/pdf/BDPfA\%20S.pdf

Naciones Unidas. (1996). 49a Asamblea Mundial de la Salud WHA49.25 Prevención de la violencia: Una prioridad de salud pública. Man. res., Vol. III (3a ed.), 1.11. http://www.who.int/violence injury prevention/resources/publications/en/ WHA4925 spa.pdf

Organización de Estados Americanos, OEA. (1994). Convención Interamericana para Prevenir, Sancionar y Erradicar la Violencia contra la Mujer. Convención de Belem do Pará. http://www.oas.org/juridico/spanish/tratados/a-61.html

Ortiz G, \& Vives C. (2012). Políticas en salud pública: Violencia contra las mujeres: el papel del sector salud en la legislación internacional. Violence against women: the role of the health sector in international legislation (English), 26, 483-489. https://doi.org/10.1016/j.gaceta.2011.09.027

Ortiz M, Forero J, Armenta A, \& Gutiérrez N. (2015). Protocolo de Vigilancia en Salud Pública. Violencia de género (p. 26). Instituto Nacional de Salud.

Ruiz P, Blanco P, \& Vives C. (2004). Violencia contra la mujer en la pareja: Determinantes y respuestas sociosanitarias. Gaceta Sanitaria, 18(5). https://www.scielosp.org/pdf/gs/2004.v18suppl2/4-12/es

Sierra S, Kerber R, \& Cook P. (2018). Suicide and Additional Homicides Associated with Intimate Partner Homicide: North Carolina 2004-2013. Journal of Urban Health, 1-7. https://doi.org/10.1007/s11524-018-0252-8

Unidad para la Atención y Reparación Integral a las Victimas. (2020, enero 1). Víctimas de conflicto armado. Reporte General. https://www.unidadvictimas.gov.co/ es/ruv/37385 
United Nations. (s. f.). Tranforming our world: The 2030 Agenda for sustainable developmen A/RES/70/1. https://sustainabledevelopment.un.org/content/documents/21252030\%20Agenda\%20for\%20Sustainable\%20Development\%20web. $\underline{\mathrm{pdf}}$

Velzeboer M, Ellsberg M, Clavel C, \& García C. (2003). La violencia contra las mujeres: Responde el sector de la salud. Organización Panamericana de la Salud, Oficina Sanitaria Panamericana, Oficina regional de la Organización Mundial de la Salud.

Vives C. (2011). Un modelo ecológico integrado para comprender la violencia contra las mujeres. Feminismo/s, 18, 291-299. 